\title{
Applications of Time-Frequency Signal Processing in Wireless Communications and Bioengineering
}

\section{Luis F. Chaparro (EURASIP Member), ${ }^{1}$ Aydın Akan (EURASIP Member), ${ }^{2}$ Syed Ismail Shah, ${ }^{3}$ and Lutfiye Durak-Ata (EURASIP Member) ${ }^{4}$}

\author{
${ }^{1}$ Department of Electrical and Computer Engineering, University of Pittsburgh, Pittsburgh, PA 15261, USA \\ ${ }^{2}$ Department of Electrical and Electronics Engineering, Istanbul University, 34320 Istanbul, Turkey \\ ${ }^{3}$ Department of Computing and Technology, Iqra University, H-9 Islamabad, Pakistan \\ ${ }^{4}$ Department of Electronics and Communications Engineering, Yildiz Technical University, 34349 Istanbul, Turkey
}

Correspondence should be addressed to Luis F. Chaparro, chaparro@ee.pitt.edu

Received 31 December 2010; Accepted 31 December 2010

Copyright (c) 2010 Luis F. Chaparro et al. This is an open access article distributed under the Creative Commons Attribution License, which permits unrestricted use, distribution, and reproduction in any medium, provided the original work is properly cited.

Time-frequency signal processing is a well-established area with applications ranging from bioengineering and wireless communications to earthquake engineering and machine monitoring. Signals in these applications are typically nonstationary and as such require joint time-frequency analysis. The objective of this special issue is to bring together theoretical results and application of time-frequency methodologies from investigators in the wireless communications and bioengineering disciplines.

Twenty outstanding articles on novel theoretical results and applications of time-frequency signal processing in wireless communications and biomedical engineering are published in this issue. The intended focus was presenting timefrequency signal processing applications to wireless communications and biomedical systems using evolutionary spectral techniques and fractional Fourier transform methods.

Five of the papers in the issue present interesting techniques on time-frequency signal processing theory. A paper in this category by L. Cohen introduces the concept of noise field in a dispersive medium. In another paper, J. Oh et al. present a method to reconstruct nonuniformly sampled signals using evolutionary Slepian transform. Z. Yin and W. Chen introduce an LFM signal detector based on the fractional Fourier transform in their paper. Another paper by A. Serbes and L. Durak-Ata discuss the eigenvectors of the discrete Fourier transform. Finally the performance analysis of high-resolution time-frequency analysis methods is presented by I. Shafi et al.
We then have four exceptional papers on the applications of time-frequency methods in communications. T. Konishi et al. introduce a new reconfigurable add/drop multiplexer using time-frequency domain processing. Channel estimation for high-mobility OFDM systems is presented for MIMO case by E. Önen et al., and for Cooperative Relaying Systems by E. Önen et al. in two papers. The final paper in this category by $\mathrm{S}$. Aldirmaz et al. discusses a new OFDMA lattice structure via toroidal waveforms.

Finally, a third group of papers in this issue are concerned with novel applications of time-frequency processing methods into biomedical engineering. M. Hassan et al. explore the pregnancy and labor contractions using uterine EMG signals. In another paper by J. Terrien et al., synchronization between EMG at different uterine locations is investigated. Time-frequency analysis of swallowing accelerometry signals is discussed by I. Orović et al. A paper is presented on data reduction for event-related potentials by S. Aviyente et al. Another paper in biomedical applications category by $\mathrm{H}$. Li et al. deals with noise and speckle reduction in Doppler blood flow spectrograms. M. Hansson-Sandsten and J. Sandberg present a method for the optimization of weighting factors for spectrogram of event-related potentials. Then cerebral hemodynamics of migraine sufferers using NIRS signals is investigated by $\mathrm{F}$. Molinari et al. A method is presented by O. Talakoub et al. to approximate time-frequency representation of biosignals with chirplets. F. Tivive et al. introduce a method to classify human gait based on radar Doppler 
spectrograms. Time-varying coherence and its application to brain functional connectivity analysis are discussed by C. Liu et al. The last paper in this group by P. Mitchell et al. deals with cross time-frequency analysis of gastrocnemius EMG signals in hypertensive and nonhypertensive subjects.

\section{Acknowledgments}

We would like to thank the authors for their interest in this special issue, their cooperation, but most of all, their outstanding contributions which are reflected so well in this issue. As usual, this special issue would not have been possible without the support of reviewers, whose comments and timely assistance assured the quality and the fast publication of the material; we also thank them. We also would like to thank the editor-in-chief for his support and give very special thanks to the editorial staff, whose help and continuous advice made our task a lot easier.

Luis F. Chaparro Aydin Akan Syed Ismail Shah

Lutfiye Durak 